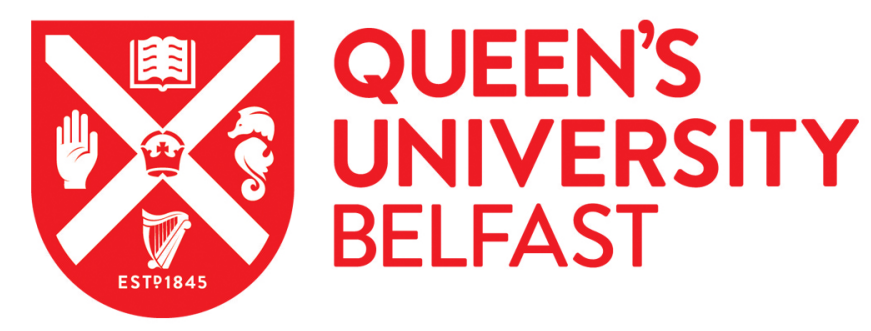

\title{
Shimmer: Implementing a Heterogeneous-Reliability DRAM Framework on a Commodity Server
}

Tovletoglou, K., Mukhanov, L., Nikolopoulos, D., \& Karakonstantis, G. (2019). Shimmer: Implementing a Heterogeneous-Reliability DRAM Framework on a Commodity Server. IEEE Computer Architecture Letters, 18(1), 26-29. https://doi.org/10.1109/LCA.2019.2893189

Published in:

IEEE Computer Architecture Letters

Document Version:

Peer reviewed version

Queen's University Belfast - Research Portal:

Link to publication record in Queen's University Belfast Research Portal

Publisher rights

(C) 2019 IEEE. This work is made available online in accordance with the publisher's policies. Please refer to any applicable terms of use of the publisher.

\section{General rights}

Copyright for the publications made accessible via the Queen's University Belfast Research Portal is retained by the author(s) and / or other copyright owners and it is a condition of accessing these publications that users recognise and abide by the legal requirements associated with these rights.

Take down policy

The Research Portal is Queen's institutional repository that provides access to Queen's research output. Every effort has been made to ensure that content in the Research Portal does not infringe any person's rights, or applicable UK laws. If you discover content in the Research Portal that you believe breaches copyright or violates any law, please contact openaccess@qub.ac.uk. 


\title{
Shimmer: Implementing a Heterogeneous-Reliability DRAM Framework on a Commodity Server
}

\author{
Konstantinos Tovletoglou, Lev Mukhanov, Dimitrios S. Nikolopoulos, and Georgios Karakonstantis \\ Queen's University Belfast, United Kingdom \{ktovletoglou01, I.mukhanov, d.nikolopoulos, g.karakonstantis\}@qub.ac.uk
}

\begin{abstract}
In this paper, we present the implementation of a heterogeneous-reliability DRAM framework, Shimmer, on a commodity server with a fully fledged OS. Shimmer enables splitting of DRAM into multiple domains with varying reliability and allocation of data depending on their criticality. Compared to existing studies which use simulators, we consider practical restrictions stemming from the real hardware and investigate methods to overcome them. In particular, we reveal that the implementation of the heterogeneous-reliability memory framework requires disabling of the hardware memory interleaving, which results in a significant degradation of the system performance. To overcome the induced performance loss, we develop a software-based interleaving. We evaluate the performance, power and energy of the server using 35 benchmarks across three memory configurations: the baseline configuration; with disabled hardware memory interleaving and Shimmer with software-based memory interleaving. Our results show that Shimmer introduces a minor $6 \%$ performance overhead, while reducing the average DRAM power by $19.9 \%$ when memory operates under relaxed refresh rate and lowered memory supply voltage. As one of our main contributions we demonstrate that a heterogeneous-reliability framework based on Shimmer can be realized on a commodity server and save $9.1 \%$ of the total processor and memory energy.
\end{abstract}

Index Terms-Power efficiency, energy saving, reliability, DRAM, critical data, heterogeneous-reliability memory, memory interleaving.

\section{INTRODUCTION}

The advent of Cloud computing and the resultant rapid increase of data is driving the aggressive scaling of DRAM for meeting the needs of higher memory density and bandwidth. As a result of the high memory demand, projections forecast that the memory subsystem will soon be responsible for more than $40 \%$ of the overall power consumption within most multicore systems [1]. However, the DRAM scaling is mainly hampered by the adoption of frequent refresh cycles and conservative voltage margins of the operating supply voltage [2] $\left(V_{D D}\right)$. Both of these parameters are selected based on the worst-case conditions to retain the stored data. Such an approach might guarantee error-free storage of data, but the incurred power and performance overheads raise doubts about its efficiency, especially as the spread in retention times increases due to worsening parametric variations [3].

Such a challenge has attracted the interest of many studies, the majority of which proposed the adoption of relaxed refresh rate $\left(T_{R E F I}\right)$ for the cells with a high retention time [3], |4]. However, recent studies [5] revealed that the retention time can dynamically change, thus limiting the effectiveness of the above schemes since the error-free properties of the 'strong' cells may not be guaranteed and threatening the operation of the system with errors. Error detection and correction codes (ECC) can be deployed to address any potential errors when the previous schemes fail $\mid[\mid$. However, ECC introduce considerable area and power overheads [6], which may negate any power gain resulted from relaxing the memory operating parameters.

Recent studies $|4|,|7|$ tried to limit such overheads by exploiting the inherent error resilient properties of various applications, allowing errors to occur. Even though an application can exhibit error resiliency for some data objects, there are data-

Manuscript submitted: 04-Oct-2018. Manuscript accepted: 24-Dec-2018. Final manuscript received: 03-Jan-2019. structures, critical data, such as the OS data $[4]$, where memory errors may result in an unacceptable output or even system crashes. Consequently, it is essential to ensure the reliable storage of the memory regions that store critical data, while the DRAM operating parameters, such as $T_{R E F I}$ and $V_{D D}$, for the remaining memory, that contains only error resilient objects, can be relaxed to save power. To enable DRAM regions with varying reliability, previous studies |7| introduced Heterogeneous-Reliability Memory (HRM) where the memory space is split into reliable regions and regions operating under relaxed parameters, that do not guarantee integrity of the data.

The potential of HRM may have been showcased on simulators $\mid 7],|8|$ or used on experimental setups for DRAM characterization neglecting the impact on the performance [9]. In fact, there are practical architectural challenges related to the implementation on a real system that have not been addressed in previous studies. Particularly, in modern DRAM subsystems, Hardware Memory Interleaving (HardInterlv) is typically being used for distributing consecutive memory accesses across multiple memory channels and thus improving performance. Such a mechanism hinders the implementation of a HRM framework as it does not allow splitting of memory into regions with varying reliability. Current solutions ignore this issue and any performance overhead introduced when HardInterlv is disabled. To the best of our knowledge, none of the existing studies have ever tried to implement a HRM on a real system with a fullyfledged OS, neither to measure the performance overhead considering these limitations nor to mitigate the incurred overhead.

Our contributions can be summarized as follows:

- We implement a cross-platform HRM framework, Shimmer, on a real commodity server with a fully-fledged OS. Shimmer enables the reliable allocation of critical data and the usage of variably-reliable memory for the rest of the data.

- We evaluate the performance overhead, that can reach as high as $128 \%$, incurred by the naive implementation of $H R M$ in which the hardware memory interleaving is disabled, using 35 benchmarks from SPEC and NAS benchmark suites.

- We propose a software-based interleaving scheme, which limits the performance overhead induced by disabling the HardInterlv down to $6 \%$.

- We demonstrate for the first time that HRM enables $9.1 \%$ energy savings on a real system when the variably-reliable memory domain allocates data in DRAM operating under relaxed $V_{D D}$ and $T_{R E F I}$.

The rest of the paper is organized as follows. Section 2 describes the DRAM background and the challenges, while Section 3 presents our proposed framework. Section 4 analyses the evaluation results. Finally, conclusions are drawn in Section 5

\section{DRAM Background and Challenges}

A main memory subsystem based on DRAM is organized into memory channel units (MCUs) supporting a number of DRAM modules. Each Dual In-line Memory Module (DIMM) consists of ranks, each of which houses a number of banks that are twodimensional arrays of cells. 
To improve the memory performance, modern servers implement HardInterlv [10]. Interleaving allows the uniform distribution of consecutive accesses across different MCUs, maximizing the memory bandwidth. Figure 19 illustrates how physical memory addresses are distributed across the MCUs when HardInterlv is enabled. We see that the physical addresses of

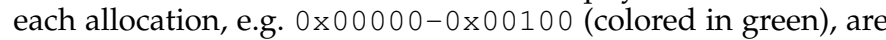
spread uniformly across all MCUs. As a result, all the accesses to these addresses will be distributed in the same way, that allows the parallel exploitation of these channels.

Challenges. HardInterlv poses a challenge to the implementation of HRM since all data is distributed across MCUs when it is enabled. Thereby, critical data would be also distributed across all MCUs, even on the ones that are configured with relaxed memory parameters. Thus, the integrity of critical data may be compromised which may lead to system crashes. To address this challenge, each MCU should have a distinguishable address space, which implies that HardInterlv must be disabled.

However, the configuration where HardInterlv is disabled (NonInterlv) may induce a performance degradation for memory-bound applications. In fact, our evaluation of the NonInterlv configuration showed that such an overhead can reach up to $128 \%$, as we will discuss later in the paper. Figure $1 \mathrm{p}$ illustrates how the physical addresses are distributed across MCUs for the NonInterlv configuration. Specifically, we see that all the

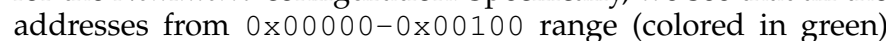
are assigned to a single MCU. Thus, the memory bandwidth in this case will be limited by the maximum bandwidth of one MCU compared to four MCUs when Hardinterlv is enabled.

\section{Proposed hrm Framework}

In this section, we demonstrate our approach on mitigating the performance overheads introduced by disabling HardInterlv in the naive HRM scheme and present its implementation.

\subsection{Software-based Memory Interleaving}

Operating systems utilize virtual addresses for data allocation, which are translated to physical addresses when the accesses are forwarded to the main memory. The MCU translates the requested addresses based on an interleaving function to the location in a specific DIMM. Note that after disabling HardInterlv, OS handles the allocation process and can use bandwidth from multiple MCUs by allocating pages across the MCUs and utilizing them in sequence. This way, we still can enable memory interleaving at the software level by mapping the continuous virtual address space to the segments of physical addresses corresponding to different MCUs. We call such a scheme Software-based Memory Interleaving (SoftInterlv). SoftInterlv allows on-the-fly selection of the interleaving scheme and the number of MCUs that will be interleaved for each allocation, in order to allow a better usage of the memory bandwidth. However, even though SoftInterlv can mitigate the performance overhead of disabling HardInterlv, there are other systems that may be affected and exhibit a performance degradation, such as memory prefetchers. Figure 11 presents an example where HardInterlv is disabled and consecutive virtual addresses, $0 \times 00000-0 \times 00100$ (colored in green), are mapped to memory from the same MCU. Furthermore, in Figure 1 1 , we see that when we enable SoftInterlv for the addresses from $0 \times 10000-0 \times 10100$ range (colored in red), the allocation is distributed across 3 MCUs, which allows increasing the memory bandwidth as the memory accesses are handled in parallel.

\subsection{Implementation}

Server Platform. To enable our study, we develop the NonInterlv configuration and Shimmer on a commodity server featuring the AppliedMicro X-Gene 2 [11] System-on-a-Chip (SoC), which hardware specification is provided in Table 1 All mem-



Fig. 1: Examples of allocation on a system a) in which HardInterlv is enabled; $b$ ) in which HardInterlv is disabled and c) in which a SoftInterlv policy is used for memory channels 1-3.

ory operations in X-Gene 2 are handled by $4 M C U_{s}$, which are divided in 2 groups of Memory Controller Bridges (MCBs). Each MCU can be populated with up to 2 DDR3 DIMMs running at $1866 \mathrm{MT} / \mathrm{s}$. In our setup, we use 4 Micron DDR3 8GB DIMMs [12], one for each MCU.

The X-Gene 2 server features a dedicated processor to enable power management, monitoring of sensors and configuration of system parameters. Those parameters include MCU initialization and memory operating parameters (such as $T_{R E F I}$ and $\left.V_{D D}\right)$. During the MCU initialization, we can define the level of HardInterlv, either across all 4 MCUs or across the 2 MCUs of each MCB or completely disable it. The default configuration of $\mathrm{X}$-Gene 2 is to have HardInterlv enabled across all MCUs.

Shimmer can be implemented in any server system that has the capability of modifying the memory operating parameters, such as $T_{R E F I}$ and $V_{D D}$. Furthermore, the server needs to have separate memory address space for each memory channel. For example, the Intel ${ }^{\circledR}$ Xeon processors [13] expose the parameter of $T_{R E F I}$ and the configuration of the memory interleaving mode between sockets and memory channels. While for tuning the $V_{D D}$ of the memory, the motherboard needs to be equipped with adjustable voltage regulators.

On top of our system, we run CentOS 7 with the Linux kernel 4.11 for ARMv8, in which we apply our changes to implement Shimmer, as described in the following sections.

Linux Kernel Modifications. We extend the interface of Non-Uniform Memory Access (NUMA) domains in Linux OS to enable HRM. Systems with multiple sockets may have varying memory access time depending on which socket the DIMM accessed by a processor belongs to. In such systems, a NUMA domain is a group of DIMMs connected to the same socket. In our study, we use fake NUMA domains and bind each of them with a separate MCU, even though the memory access time is identical for each X-Gene 2 core.

We introduce 4 fake NUMA domains and assign each one

TABLE 1: Hardware platform specifications.

\begin{tabular}{lc}
\hline Description & Value \\
\hline System & AppliedMicro X-Gene 2 \\
Architecture & ARMv8 \\
Core Frequency & $2.4 \mathrm{GHz}$ \\
Number of Cores & 8 \\
Last Level Cache & $8 \mathrm{MB}$ (shared) \\
\hline \# of Memory Controllers & 4 \\
Memory Size & 8 GB/MCU, Total 32 GB \\
Memory Type & DDR3-1866, SECDED ECC \\
Memory Characteristics & 2 rank/MCU, 8 banks/rank, \\
& 64 K rows/bank, 64 B cache line \\
\hline
\end{tabular}




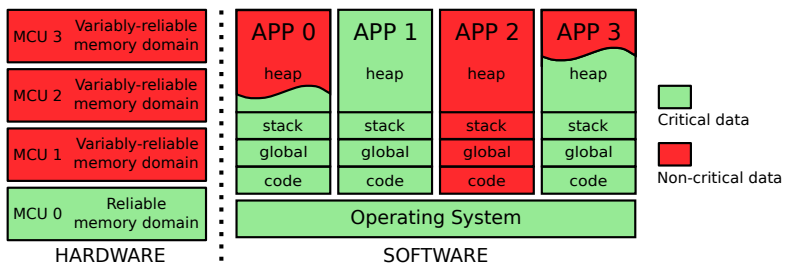

Fig. 2: Hardware setup for the variably-reliable memory and possible allocations of applications in the memory.

to the memory address range of one MCU. By default, applications allocate data using the first NUMA domain that we use as the Reliable Memory (RelMem) domain. This domain is assigned to the MCU which uses the nominal memory operating parameters. The rest of the domains, Variably-reliable Memory (VarMem) domains, are assigned to MCUs in which DRAM reliability may vary as the memory operating parameters can be relaxed. Our framework can be adopted in most servers that have separate memory address space for each memory channel or that memory channel interleaving can be disabled. Shimmer can be also used in multi-socket systems since it does not restrict the use of the NUMA interface.

Allocation Interface of HRM. By default, the Linux kernel and every application, which uses the standard system functions, allocate data in RelMem. Furthermore, Shimmer allows the user to control the memory allocations for an application through the NUMA interface using the numactl command. Particularly, the parameter --membind can be passed to numact 1 to define the NUMA memory domain that will be used for data allocation. In Figure 2 $A P P 1$ and $A P P 2$ are examples of such an allocation, where all application data is assigned to either the RelMem or the VarMem domain.

We enable SoftInterlv of the memory in Shimmer by extending the numactl command with a new parameter --interleave across the NUMA memory domains. In such a way, we can utilize the memory channel parallelism and exploit the bandwidth of multiple MCUs, similarly to HardInterlv. We enable SoftInterlv using 3 interleaved MCUs, which can be assigned to the VarMem domain, so that the remaining MCU is used for the RelMem domain to store critical data.

To choose the reliability domain for a specific data allocation within an application, we extend the standard memory allocation interface, i.e. malloc, with a new interface, numa_alloc_onnode, that allocates data in a particular NUMA domain. The programmer can allocate non-critical data structures in the VarMem domain using this interface by modifying the source code of an application. Figure 2 illustrates such an allocation, where applications $A P P 0$ and $A P P 3$ allocate a part of the application's heap data in the VarMem domain.

The programmer must analyze the workloads to identify the criticality of the data structures and therefore select in which reliability domain to allocate the data. Recent research work [14] presented an automated technique to identify the resilience of data structures through analytical models, however, analysis of the data criticality is out of the scope of this paper.

Error Detection and Correction. The system reports all errors, either correctable or uncorrectable, detected by ECC and provides information about the memory location where the error occur: specifically, the MCU, rank, bank, row and column. Importantly, in our experiments with relaxed operating parameters set for the VarMem domain, all of the errors were corrected by the available ECC. This observation implies that the execution of benchmarks is not affected by memory errors in our experiments. Nonetheless, uncorrectable errors may occur in the VarMem domain, e.g. under high DRAM temperatures, and thus, each application allocating data in this domain should use a fault tolerance technique to handle such errors. We note that research of such techniques is also beyond the scope of our study.

\section{Experimental Results}

In this section, we evaluate the performance, power and energy of the server using a variety of benchmarks across three memory configurations: $i$ ) the default HardInterlv configuration; ii) the NonInterlv configuration and iii) Shimmer.

\subsection{Workloads}

We choose a set of workloads, ranging from micro-benchmarks that are typically used for evaluation of the memory bandwidth to real-world applications. These workloads stress the processor and memory in a different way, which allows us to investigate systematically the impact of the memory configurations on performance, power and energy.

We execute LMbench [15] to identify any deficiency in utilization of the memory bandwidth. From SPEC CPU2006 [16], we perform experiments with 28 of the benchmarks using the ref data inputs, while running a single-threaded instance of the benchmark for each core in parallel. We also run 7 benchmarks from NAS Parallel Benchmarks [17] using the C input class and the OpenMP programming model. In all our experiments, we use 8 cores for parallel execution.

Note that we allocate all application data from both benchmark suites in the VarMem domain. For the NonInterlv system and Shimmer, the VarMem domain is configured to utilize 3 MCUs (1, 2, 3). Meanwhile, OS data is allocated in the RelMem domain, i.e. MCU 0, which operates under nominal parameters.

\subsection{Performance Evaluation}

The disabling of HardInterlv is accompanied with a performance degradation of the entire system, since the memory accesses are not distributed across all MCUs. To measure the degradation, we run $L M b e n c h$ and use $8 G B$ of memory, in order to restrict the allocation within a single MCU. The maximum memory bandwidth of the system with enabled Hardinterlv is measured at $22.3 \mathrm{~GB} / \mathrm{s}$. Once we disable HardInterlv and enable Shimmer, the bandwidth reaches $16.4 \mathrm{~GB} / \mathrm{s}$. The $27 \%$ drop is explained by the fact that only three MCUs are used instead of four. While the bandwidth per MCU drops slightly from $5.57 \mathrm{~GB} / \mathrm{s}$ when HardInterlv is enabled to $5.46 \mathrm{~GB} / \mathrm{s}$ for Shimmer.

Figure 3 presents the performance overhead introduced by the NonInterlv system and Shimmer compared to the baseline configuration for the SPEC and NAS benchmarks. We calculate this overhead by measuring the execution time of each benchmark for the two configurations normalized to the execution times obtained for the default server configuration. Note that, in this figure, we use benchmark identification numbers instead of the whole SPEC benchmark names. We see that the NonInterlv configuration introduces an average performance overhead of $49.39 \%$, which is expected as the memory bandwidth is not

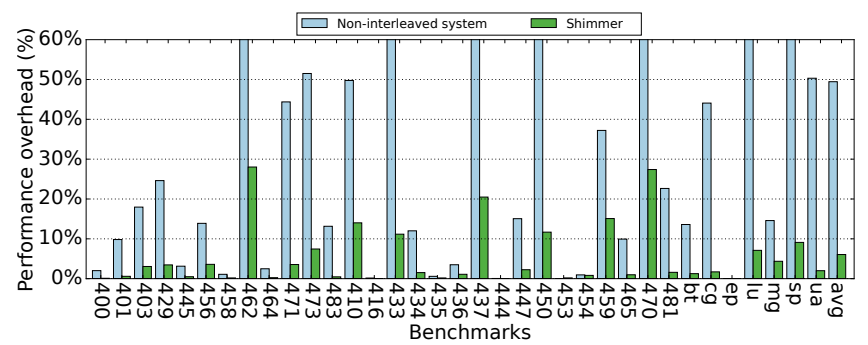

Fig. 3: Performance overhead manifested on the NonInterlv system and Shimmer compared to the HardInterlv system for the SPEC and NAS benchmarks. 

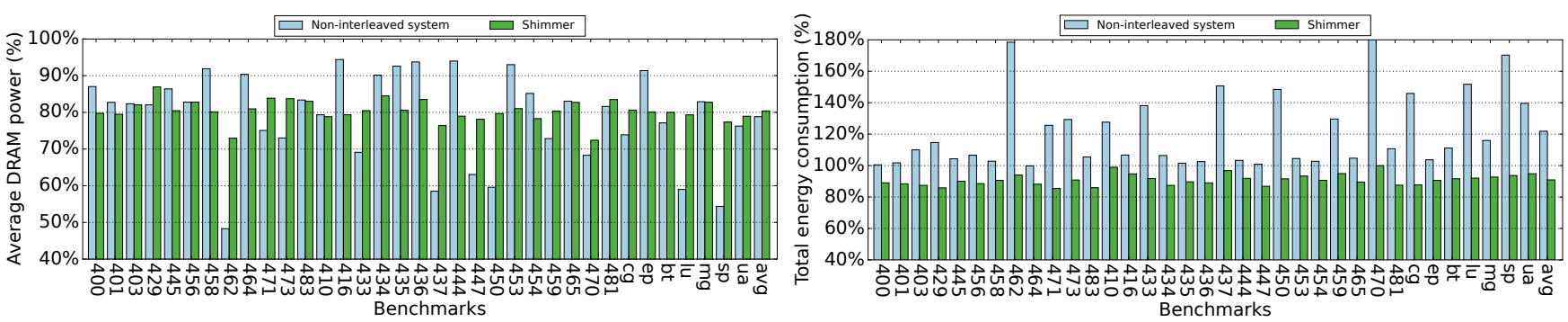

Fig. 4: a) The average DRAM power and $b$ ) the total energy consumption of the NonInterlv system and Shimmer normalized to the power and energy of the HardInterlv system across the SPEC and NAS benchmarks.

fully exploited. However, in the case of Shimmer, the average overhead decreases down to $6 \%$. We also observe that the performance overhead varies across benchmarks, while the highest overhead is manifested for the 462 . libquantum and $470.1 \mathrm{bm}$ benchmarks, $111 \%$ and $128 \%$ respectively for the NonInterlv configuration. Notably, the same benchmarks have also the highest overhead for Shimmer, which is about $28 \%$.

\subsection{Power and Energy Evaluation}

To achieve the maximum power gain, we configure the $3 \mathrm{MCUs}$ of the VarMem domain using the least reliable settings for $V_{D D}$ and $T_{R E F I}$. Note that our platform allows lowering of $V_{D D}$ on the DIMMs associated with the second MCB (MCU 2 and 3) only, while we are able to relax $T_{R E F I}$ for MCU 1, 2 and 3. We set the $V_{D D}$ to the minimum voltage specified by the DIMM's datasheet [12], i.e. we reduce $V_{D D}$ from $1.5 \mathrm{~V}$ to $1.425 \mathrm{~V}(5 \%$ reduction), and increase $T_{R E F I}$ from the nominal $64 \mathrm{~ms}$ to $2.283 s(35 \times$ increase $)$ that is the maximum allowed $T_{R E F I}$ in the X-Gene 2 platform. We measure power of DRAM and SoC using real voltage and current sensors that are integrated with voltage regulators on the X-Gene 2 motherboard.

Figure $4 \mathrm{a}$ shows the memory power consumption for the NonInterlv system and Shimmer, operating under relaxed DRAM parameters, normalized to the power measured for the baseline configuration under nominal $V_{D D}$ and $T_{R E F I}$. We see that the highest DRAM power reduction is achieved for the NonInterlv system, which is about $23 \%$. While we can lower the average DRAM power consumption by $19.9 \%$ when Shimmer is enabled. Notably, in the case of the 462 . Iibquantum benchmark, which has the highest power consumption, Shimmer enables $27.1 \%$ DRAM power savings that is the maximum power reduction achieved among all benchmarks.

We also evaluate the total energy consumed by the server when running the SPEC and NAS benchmarks. We calculate the total energy consumption of the system $\left(E_{\text {total }}\right)$ by integrating the DRAM and SoC power $\left(P_{\text {total }}\right)$ over the duration $(T)$ for each benchmark: $E_{\text {total }}=\int_{0}^{T} P_{\text {total }}(t) d t$. Figure $4 \mathrm{~b}$ shows the total energy for the NonInterlv system and Shimmer normalized to the energy consumed by the server using the default configuration when DRAM operates under nominal $V_{D D}$ and $T_{R E F I}$. We see that in the case of the NonInterlv system, the average energy consumption grows by $21.8 \%$, and no benchmark achieves any energy savings. By contrast, Shimmer achieves an average energy reduction of $9.1 \%$. Moreover, only 3 benchmarks, i.e. $470.1 \mathrm{bm}, 410$.bwaves and 444 . namd, have an energy reduction less than $5 \%$ using Shimmer.

\section{Conclusion}

In this paper, we present a cross-platform heterogeneousreliability DRAM framework, Shimmer, implemented for the first time on a commercial server allowing us to obtain energy savings. We demonstrate that one of the main obstacles that hampers the implementation of heterogeneous-reliability memory on a real server is the hardware memory interleaving, which has been neglected by previous studies. We systematically investigate the performance overhead incurred when the interleaving is disabled, and we implement a software-based interleaving technique through the NUMA interface to reduce the incurred overhead significantly. Our results show that even though Shimmer introduces an average performance overhead of $6 \%$, it enables the operation of parts of the DRAM under relaxed parameters and reduces the DRAM power by $19.9 \%$ on average. Overall, Shimmer allows us to save $9.1 \%$ of the system energy when DRAM operates under relaxed parameters, while allocating data in the error-free domain of memory, and thus, offering protection of the critical data, including the OS data.

\section{ACKNOWLEDGEMENTS}

This work is funded by the H2020 Programme of the European Union under grant no. 688540 (the UniServer Project).

\section{REFERENCES}

[1] B. Giridhar et al., "Exploring dram organizations for energyefficient and resilient exascale memories," in Proc. Int. Conf. on High Perform. Comp., Netw., Storage and Analysis, 2013, pp. 1-12.

[2] K. K. Chang et al., "Understanding reduced-voltage operation in modern dram devices: Experimental characterization, analysis, and mechanisms," in Proc. ACM SIGMETRICS, 2017, pp. 52-52.

[3] J. Liu et al., "Raidr: Retention-aware intelligent dram refresh," in Proc. Int. Symposium on Computer Architecture, 2012, pp. 1-12.

[4] A. Raha et al., "Quality-aware data allocation in approximate dram," in Proc. Int. Conf. on Compilers, Architecture and Synthesis for Embedded Systems, 2015, pp. 89-98.

[5] M. K. Qureshi et al., "Avatar: A variable-retention-time (vrt) aware refresh for dram systems," in Proc. Int. Conf. on Dependable Sys. and Netw., 2015, pp. 427-437.

[6] P. J. Nair et al., "Archshield: Architectural framework for assisting dram scaling by tolerating high error rates," in Proc. Int. Symposium on Computer Architecture, 2013, pp. 72-83.

[7] S. Liu et al., "Flikker: Saving dram refresh-power through critical data partitioning," SIGARCH Comp. Archit. News, p. 213, 2011.

[8] M. Jung et al., "Omitting refresh: A case study for commodity and wide i/o drams," in Proc. Int. Symp. on Mem. Sys., 2015, pp. 85-91.

[9] C. Chalios et al., "Dare: Data-access aware refresh via spatialtemporal application resilience on commodity servers," Int. Journal of High Perf. Comp. Applic., vol. 32, no. 1, pp. 74-88, 2018.

[10] Z. Zhang et al., "A permutation-based page interleaving scheme to reduce row-buffer conflicts and exploit data locality," in Proc. ACM/IEEE Int. Symp. on Microarchitecture, 2000, pp. 32-41.

[11] G. Singh et al., "AppliedMicro X-Gene 2," in IEEE Hot Chips 26 Symp., 2014, pp. 1-24.

[12] Micron Technology, "MT18JSF1G72AZ-1G9 - 8GB," 2015.

[13] Intel, "Intel xeon processor e5-1600/2400/2600/4600 (e5-product family) product families datasheet, volume two," Intel, 2012.

[14] V. Vassiliadis et al., "Towards automatic significance analysis for approximate computing," in Proc. Int. Symposium on Code Generation and Optimization, 2016, pp. 182-193.

[15] L. McVoy et al., "Lmbench: Portable tools for performance analysis," in Proc. USENIX Annual Technical Conf., 1996, pp. 279-294.

[16] J. L. Henning, "Spec cpu2006 benchmark descriptions," SIGARCH Comput. Archit. News, vol. 34, pp. 1-17, 2006.

[17] D. H. Bailey et al., "Nas parallel benchmark results," in Proc. ACM/IEEE Conf. on Supercomputing, 1992, pp. 386-393. 